\title{
Epidemiology of trauma in Venezuelan immigrants in Bucaramanga, Colombia: A look at a neglected phenomenon
}

\section{Epidemiologia del trauma en inmigrantes venezolanos en Bucaramanga, Colombia: Una mirada a un fenómeno desatendido}

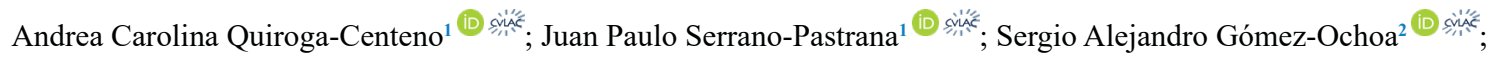 \\ * caroline_aqc@hotmail.com.
}

Suggested citation: Quiroga Centeno AC, Serrano Pastrana JP, Gómez Ochoa SA. Epidemiology of trauma in Venezuelan immigrants in Bucaramanga, Colombia: A look at a neglected phenomenon. Salud UIS. 2022; e22019. doi: https://oi. org/10.18273/saluduis.54.e:22019 (c) (1)

Dear Editor,

In recent years, the immigration of Venezuelan citizens to the Republic of Colombia has increased ${ }^{1}$. Although some research has shown a difference in the presentation of medical conditions in these individuals, there are still no studies evaluating the epidemiology of trauma in this population ${ }^{2,3}$. The objective of the present study was to compare the characteristics of trauma in the native Colombian population versus the Venezuelan immigrant population using the Institutional Trauma Registry of the Hospital Universitario de Santander (RITHUS) in its first year of implementation.

A prospective cohort study was conducted. All patients with external-cause injuries managed in the Adult Emergency Department of the Hospital Universitario de Santander (HUS) from September 1, 2020, to July 31, 2021, were included. Categorical variables were expressed as absolute values and proportions, while continuous variables were reported as medians and quartiles 1 and 3 . The $\mathrm{X}^{2}$ test was used to evaluate differences between categorical variables, and Student's t-test and Mann Whitney U test for quantitative variables, according to their distribution. Finally, a logistic regression model was used to evaluate the presence of an association between Venezuelan migrant status and the risk of in-hospital mortality associated with trauma. An $\alpha$ level of 0.05 (bilateral) was considered statistically significant. The data set was constructed and analyzed using Stata/MP, version 16.0 (StataCorp, College Station, Texas).

\footnotetext{
${ }^{1}$ Universidad Industrial de Santander, Bucaramanga, Colombia.

${ }^{2}$ University of Bern, Bern, Switzerland.
} 
Of the total of 3,114 patients included, 416 (13\%) were of Venezuelan nationality. Their median age was 26 years, which was significantly lower than that of the national patients $(32$ years $)(p<0.001)$. In addition, Venezuelan immigrants consulted more frequently by their own means (i.e., on foot, by private vehicle or taxi) compared to Colombians $(80.9 \%$ vs. $72.4 \%, p=0.036)$. The median prehospital time of these patients was 2.4 hours $(\mathrm{Q} 1: 1.1$; $\mathrm{Q} 3: 15.6)$, which was not significantly different from the Colombian counterpart ( $\mathrm{P}$-value $=0.277$ ). No differences were found regarding sex, type of admission (referred or direct) or type of violence (physical, psychological or sexual abuse). Additionally, the Venezuelan patients had a lower consumption of psychoactive substances associated with trauma $(\mathrm{P}<0.001)$ (Table 1$)$.

Table 1. Baseline characteristics of patients presenting to the emergency department with external-cause injuries, stratified by immigrant status.

\begin{tabular}{|c|c|c|c|c|}
\hline Variables & $\begin{array}{c}\begin{array}{c}\text { Colombian } \\
\text { patients }\end{array} \\
\mathrm{N}=\mathbf{2 6 9 8}\end{array}$ & $\begin{array}{l}\text { Venezuelan patients } \\
\qquad \mathrm{N}=\mathbf{4 1 6}\end{array}$ & $\begin{array}{c}\text { Total } \\
\text { N=3114 }\end{array}$ & P-value \\
\hline Age & $32(24 ; 47)$ & $26(21 ; 33.5)$ & $31(23 ; 46)$ & $<0.001$ \\
\hline Males & $2120(78.6)$ & $312(75.0)$ & $2432(78.1)$ & 0.101 \\
\hline \multicolumn{5}{|l|}{ Pre-hospital transport } \\
\hline Own means & $1304(48.3)$ & $237(57)$ & $1541(49.5)$ & \multirow{5}{*}{0.041} \\
\hline Ambulance & $1074(39.8)$ & $141(33.9)$ & $1215(39.0)$ & \\
\hline Police & $220(8.2)$ & $25(6.0)$ & $245(7.9)$ & \\
\hline Others (civil defense, firefighters) & $67(2.5)$ & $8(1.9)$ & $75(2.4)$ & \\
\hline Missing data & $33(1.2)$ & $5(1.2)$ & $38(1.2)$ & \\
\hline \multicolumn{5}{|l|}{ Intentionality of injury } \\
\hline Accidental & $1550(57.5)$ & $263(63.2)$ & $1813(58.2)$ & \multirow{5}{*}{0.141} \\
\hline Physical aggression & $956(35.4)$ & $126(30.3)$ & $1082(34.8)$ & \\
\hline Self-inflicted injury & $38(1.4)$ & $4(1)$ & $42(1.4)$ & \\
\hline Others & $52(1.9)$ & $7(1.7)$ & $59(1.9)$ & \\
\hline Missing data & $102(3.8)$ & $16(3.8)$ & $118(3.7)$ & \\
\hline $\begin{array}{l}\text { Use of psychoactive } \\
\text { substances }\end{array}$ & $411(15.4)$ & $31(7.5)$ & $442(14.3)$ & $<0.001$ \\
\hline
\end{tabular}

This table contains the $\%$ for categorical variables and the median (first and third quartiles) for continuous variables.

The most frequently observed mechanism of trauma was penetrating trauma $(41.8 \% ; \mathrm{n}=1,302)$, with stab wounds being the most frequent $(24.9 \% ; \mathrm{n}=776)$. On the other hand, in 1,299 patients $(41.7 \%)$, blunt trauma was the only mechanism of injury, with falls from height $(18.9 \% ; n=587)$, and traffic accidents $(16.3 \% ; n=508)$ being the most common types of trauma in this group. Finally, mixed trauma (concomitant penetrating and blunt) was recorded in 207 patients $(6.6 \%)$. No significant differences by nationality were observed in terms of the mechanism of injury. However, Venezuelan migrants had a significantly higher incidence of blunt weapon injuries than Colombian patients (12.3\% vs. $9 \% ; \mathrm{p}=0.032$ ), this difference being independent of sex, age and intentionality of injury (OR $1.51 ; 95 \%$ CI 1.08-2.12). No other significant differences were observed by type of injury. Regarding trauma severity, the mean Revised Trauma Score (RTS) in Venezuelan migrants was significantly higher compared to Colombian patients (7.74 \pm 0.55 vs. $7.62 \pm 0.86 ; \mathrm{p}=0.001)$. The median hospital stay for the entire cohort was two days (Q1: 0; Q3: 4), with no significant differences by nationality $(\mathrm{p}=0.144)$. Finally, the risk of mortality was similar in both groups after adjusting for age, sex, intention of injury and mechanism of trauma (OR 0.34; 95\% CI 0.11-1.09). 
The results of the present study highlight the important disparities in the epidemiology of trauma according to migrant status in Colombia, observing similarities with what has been reported in other studies conducted internationally in refugee populations. First, the finding of a significantly lower median age in Venezuelan migrants compared to the native population is in line with what has been reported in the literature in the field of trauma ${ }^{4,5}$.

On the other hand, there were no significant differences in the mechanism of trauma or the intentionality of the injury between the two populations. This finding is in opposition to what has been reported in other studies, which describe a higher risk of stab or firearm injuries in the migrant population ${ }^{4,7}$. Furthermore, differences in trauma severity in migrant populations have been widely debated in the literature, with divergent results regarding injury severity according to migrant status. These differences can potentially be associated with diverse cultural and social contexts, which limits the extrapolation of results from individual studies ${ }^{4,7-9}$.

In conclusion, Venezuelan migrants admitted to the emergency department for an external cause injury present a significantly different sociodemographic and clinical profile than Colombian nationals, which highlights the need to further evaluate the social and economic conditions behind these findings, as well as to generate public health policies that consider migrant populations.

\section{Epidemiologia del trauma en inmigrantes venezolanos en Bucaramanga, Colombia: Una mirada a un fenómeno desatendido}

\section{Sr. Editor:}

En los últimos años se ha incrementado la inmigración de ciudadanos venezolanos a la República de Colombia ${ }^{1}$. Aunque algunas investigaciones han mostrado una diferencia en la presentación de condiciones médicas en estos individuos, aún no existen estudios que evalúen la epidemiología del trauma en esta población ${ }^{2,3}$. El objetivo del presente estudio fue comparar las características del trauma en población nacional versus la inmigrante venezolana haciendo uso del Registro Institucional de Trauma del Hospital Universitario de Santander (RITHUS) en su primer año de implementación.

Se realizó un estudio de cohorte prospectivo en el cual se incluyeron todos los pacientes con lesiones de causa externa manejados en el Servicio de Urgencias de Adultos del Hospital Universitario de Santander (HUS) desde el 1 de septiembre de 2020 hasta el 31 de julio de 2021. Las variables categóricas se expresaron como valores absolutos y proporciones, mientras que las variables continuas se reportaron como medianas y cuartiles 1 y 3 . Se utilizó la prueba de X2 para evaluar diferencias entre las variables categóricas, y la prueba t de Student y U de Mann Whitney para las variables cuantitativas, según su distribución. Finalmente, se utilizó un modelo de regresión logística para evaluar la presencia de una asociación entre el estatus de migrante venezolano y el riesgo de mortalidad intrahospitalaria asociada al trauma. Un nivel $\alpha$ de 0,05 (bilateral) se consideró estadísticamente significativo. El conjunto de datos se construyó y analizó utilizando Stata/MP, versión 16.0 (StataCorp, College Station, Texas).

Del total de 3114 pacientes incluidos, se identificó que 416 (13\%) eran de nacionalidad venezolana. Su mediana de edad fue de 26 años, la cual fue significativamente inferior a la de los pacientes nacionales ( 32 años) ( $<<0,001)$. Además, los inmigrantes venezolanos ingresaron con mayor frecuencia por sus propios medios (es decir, a pie, 
en vehículo particular o en taxi) en comparación con los colombianos $(80,9 \%$ vs $72,4 \%, p=0,036)$. La mediana del tiempo prehospitalario de estos pacientes fue de 2,4 horas (Q1:1,1; Q3:15,6), lo cual no fue significativamente diferente a la contraparte colombiana ( $\mathrm{P}$-value $=0,277)$. No se encontraron diferencias en el sexo, el tipo de ingreso (referido o directo) o el tipo de violencia (abuso físico, psicológico o sexual). Adicionalmente, los pacientes venezolanos presentaron un menor consumo de sustancias psicoactivas asociadas al trauma ( $\mathrm{p}<0,001)$ (Tabla 1).

Tabla 1. Características basales de los pacientes que acuden al servicio de urgencias con lesiones de causa externa, estratificados por su condición de inmigrantes.

\begin{tabular}{|c|c|c|c|c|}
\hline Variables & $\begin{array}{c}\begin{array}{c}\text { Pacientes } \\
\text { colombianos }\end{array} \\
\mathbf{N}=\mathbf{2 6 9 8}\end{array}$ & $\begin{array}{c}\begin{array}{c}\text { Pacientes } \\
\text { venezolanos }\end{array} \\
\mathrm{N}=416\end{array}$ & $\begin{array}{c}\text { Total } \\
\mathrm{N}=3114\end{array}$ & Valor $\mathbf{P}$ \\
\hline Edad & $32(24 ; 47)$ & $26(21 ; 33.5)$ & $31(23 ; 46)$ & $<0.001$ \\
\hline Sexo masculino & $2120(78.6)$ & $312(75.0)$ & $2432(78.1)$ & 0.101 \\
\hline \multicolumn{5}{|l|}{ Transporte prehospitalario } \\
\hline Propios medios & $1304(48.3)$ & $237(57)$ & $1541(49.5)$ & \multirow{5}{*}{0.041} \\
\hline Ambulancia & $1074(39.8)$ & $141(33.9)$ & $1215(39.0)$ & \\
\hline Policía & $220(8.2)$ & $25(6.0)$ & 245 (7.9) & \\
\hline Otros (defensa civil, bomberos) & $67(2.5)$ & $8(1.9)$ & $75(2.4)$ & \\
\hline Datos faltantes & $33(1.2)$ & $5(1.2)$ & $38(1.2)$ & \\
\hline \multicolumn{5}{|l|}{ Intencionalidad de lesión } \\
\hline Accidental & $1550(57.5)$ & $263(63.2)$ & $1813(58.2)$ & \multirow{5}{*}{0.141} \\
\hline Agresión física & $956(35.4)$ & $126(30.3)$ & $1082(34.8)$ & \\
\hline Lesión autoinfligida & $38(1.4)$ & $4(1)$ & $42(1.4)$ & \\
\hline Otros & $52(1.9)$ & $7(1.7)$ & $59(1.9)$ & \\
\hline Datos faltantes & $102(3.8)$ & $16(3.8)$ & $118(3.7)$ & \\
\hline $\begin{array}{l}\text { Consumo de sustancias } \\
\text { psicoactivas }\end{array}$ & $411(15.4)$ & $31(7.5)$ & $442(14.3)$ & $<0.001$ \\
\hline
\end{tabular}

Esta tabla contiene el \% para las variables categóricas y la mediana (primer y tercer cuartil) para las variables continuas.

El mecanismo de traumatismo más frecuentemente observado fue el trauma penetrante $(41,8 \% ; n=1302)$, siendo las lesiones por arma blanca las más frecuentes $(24,9 \% ; n=776)$. Por otro lado, en 1299 pacientes $(41,7 \%)$, el trauma contundente fue el único mecanismo de lesión, siendo las caídas de altura $(18,9 \% ; \mathrm{n}=587)$, y los accidentes de tráfico $(16,3 \% ; n=508)$ los tipos de trauma más comunes en este grupo. Por último, los traumatismos mixtos (penetrantes y contundentes concomitantes) se registraron en 207 pacientes $(6,6 \%)$. No se observaron diferencias significativas por nacionalidad en cuanto al mecanismo de la lesión. Sin embargo, los migrantes venezolanos tuvieron una incidencia significativamente mayor de lesiones por arma cortocontundente que los pacientes colombianos $(12,3 \%$ vs. $9 \%$; $=0,032)$, siendo esta diferencia independiente del sexo, la edad y la intencionalidad de la lesión (OR 1,51 ; IC $95 \% 1,08-2,12$ ). No se observaron otras diferencias significativas por tipo de lesión. En cuanto a la severidad del trauma, la media del Revised Trauma Score (RTS) en los migrantes venezolanos fue significativamente mayor en comparación con los pacientes colombianos $(7,74 \pm 0,55$ vs $7,62 \pm 0,86 ; \mathrm{p}=0,001)$. La mediana de la estancia hospitalaria para toda la cohorte fue de dos días (Q1: 0; Q3: 4), sin que se observaran diferencias significativas por nacionalidad $(\mathrm{p}=0,144)$. Finalmente, el riesgo de mortalidad fue similar en ambos grupos tras ajustar por edad, sexo, intención de la lesión y mecanismo del trauma (OR 0,34; IC 95 \% 0,11-1,09). 
Los resultados del presente estudio ponen de manifiesto las importantes disparidades en la epidemiología del trauma según la condición de migrante en Colombia, observándose similaridades con lo reportado en otros estudios realizados a nivel internacional en poblaciones de refugiados. En primera medida, el hallazgo de una mediana de edad significativamente menor en los migrantes venezolanos en comparación con la población nativa está en línea con lo reportado en la literatura en el ámbito del trauma ${ }^{4,5}$.

Por otra parte, no hubo diferencias significativas respecto al mecanismo del trauma ni la intencionalidad de la lesión entre las dos poblaciones. Este hallazgo se encuentra en oposición a lo reportado en otros estudios, en los cuales se describe un mayor riesgo de lesiones por arma blanca o de fuego en población migrante ${ }^{4,7}$. Además, las diferencias en la severidad del trauma en población migrante han sido ampliamente debatidas en la literatura, con resultados divergentes respecto a la gravedad de las lesiones de acuerdo con el estatus de migrante. Estas diferencias pueden asociarse potencialmente a los diversos contextos culturales y sociales, lo cual limita la extrapolación de los resultados de estudios individuales ${ }^{4,7-9}$.

En conclusión, los migrantes venezolanos que ingresan al servicio de urgencias por una lesión de causa externa presentan un perfil sociodemográfico y clínico significativamente diferente al de los nacionales colombianos, lo que pone de manifiesto la necesidad de seguir evaluando las condiciones sociales y económicas que están detrás de estos hallazgos, así como de generar políticas de salud pública que consideren a las poblaciones migrantes.

\section{References}

1. The UN Refugee Agency (UNHCR). UNHCR - Venezuela situation. 2021. https://www.unhcr.org/venezuelaemergency.html.

2. Luna Orozco K, Fernández-Niño JA. Migración venezolana en Colombia: retos en Salud Pública. Rev Univ Ind Santander Salud. 2018; 50(1): 5-6.

3. Zambrano-Barragán P, Ramírez Hernández S, Freier LF, Luzes M, Sobczyk R, Rodríguez A, et al. The impact of COVID-19 on Venezuelan migrants' access to health: A qualitative study in Colombian and Peruvian cities. J Migr Health. 2021; 3: 100029. doi: https://doi.org/10.1016/j.jmh.2020.100029

4. Al-Hajj S, Chahrour MA, Nasrallah AA, Hamed L, Pike I. Physical trauma and injury: A multi-center study comparing local residents and refugees in Lebanon. J Glob Health. 2021; 11: 17001. doi: http://dx.doi. org/10.7189/jogh.11.17001

5. Saunders NR, Macpherson A, Guan J, Guttmann A. Unintentional injuries among refugee and immigrant children and youth in Ontario, Canada: a population-based cross-sectional study. Inj Prev J Int Soc Child Adolesc Inj Prev. 2018; 24(5): 337-343. doi: http://dx.doi.org/10.1136/injuryprev-2016-042276

6. Miller LC, Timouri M, Wijnker J, Schaller JG. Afghan refugee children and mothers. Arch Pediatr Adolesc Med. 1994; 148(7): 704-708. doi: https://doi.org/10.1001/archpedi.1994.02170070042007

7. Perlman A, Radomislensky I, Israel Trauma Group, Peleg K. Injury patterns among illegal migrants from Africa in Israel. J Immigr Minor Health. 2015; 17(4): 1163-1168. doi: https://doi.org/10.1007/s10903-014-0068-X

8. Norredam M, Olsbjerg M, Petersen JH, Laursen B, Krasnik A. Are there differences in injury mortality among refugees and immigrants compared with native-born? Inj Prev J Int Soc Child Adolesc Inj Prev. 2013; 19(2): 100-105. doi: http://dx.doi.org/10.1136/injuryprev-2012-040336

9. Tsai YC, Wu NC, Su HC, Hsu CC, Guo H-R, Chen K-T. Differences in injury and trauma management between migrant workers and citizens. Medicine (Baltimore). 2020; 99(31): e21553. doi: http://dx.doi.org/ 10.1097/ MD.0000000000021553 